Philosophie ANTIQUE

\section{Philosophie antique}

Problèmes, Renaissances, Usages

$20 \mid 2020$

Nouvelles figures de Socrate

\title{
Livio RosSETTI, Convincere Socrate
}

Bologna, Diogene Multimedia, 2017 (Autobiografie e biografie), 110 p., ISBN 978-88-9363-080-1

\section{Fulvia De Luise}

\section{(2) OpenEdition}

\section{Journals}

\section{Edizione digitale}

URL: https://journals.openedition.org/philosant/1977

DOI: 10.4000/philosant.1977

ISSN: 2648-2789

\section{Editore}

Éditions Vrin

\section{Edizione cartacea}

Data di pubblicazione: 31 octobre 2020

Paginazione: 282-285

ISBN: 978-2-7116-2977-0

ISSN: $1634-4561$

\section{Notizia bibliografica digitale}

Fulvia De Luise, «Livio Rossetтı, Convincere Socrate », Philosophie antique [En ligne], 20 | 2020, mis en ligne le 10 mai 2019, consulté le 06 décembre 2022. URL : http://journals.openedition.org/philosant/ 1977 ; DOI : https://doi.org/10.4000/philosant.1977

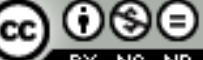

Creative Commons - Attribution - Pas d'Utilisation Commerciale - Pas de Modification 4.0 International - CC BY-NC-ND 4.0

https://creativecommons.org/licenses/by-nc-nd/4.0/ 
pieds fermement sur la terre : il montre, en fait, que l'objectif fondamental de Parménide est de nous parler de notre monde. En réévaluant l'importance de l'expérience et des observations dans la formulation des doctrines de Parménide, Rossetti remet en question de manière radicale une tradition qui, à partir de Platon, a concentré son attention sur une doctrine - celle de l'être - qui apparaît totalement a priori. Je pense qu'il s'agit d'un défi qui mérite d'être relevé.

Guido Calenda

Livio Rossetti, Convincere Socrate, Bologna, Diogene Multimedia, 2017 (Autobiografie e biografie), 110 p., ISBN 978-88-9363-080-1.

La scrittura letteraria è sempre in qualche modo riscrittura. E per apprezzarne l'originalità occorre immergersi nel labirinto di citazioni di cui un testo d'autore abitualmente si compone. Ciò è tanto più vero nel caso della letteratura socratica, in cui tutto ciò che sappiamo di Socrate, come oggetto reale della rappresentazione, è appassionata trascrizione di una indimenticabile esperienza collettiva, riscritta a molte mani e in sé irrappresentabile. Di qui la tentazione (ricorrente nella tradizione di cui siamo eredi) di entrare nella scena socratica per riviverla e per far rivivere, con nuove domande e nuove indagini, una storia divenuta canonica soprattutto nel suo drammatico esito finale: la morte di Socrate, la condanna di un uomo che non chiede venia per ciò che è stato e non si sottrae con l'inganno a un verdetto ingiusto.

Dobbiamo alla suggestione di questo fatto, che impressionò potentemente i contemporanei, la nascita stessa della scrittura filosofica, che Platone ha inaugurato, rendendola inseparabile dal mito del filosofo che paga con la vita la sua coerenza intellettuale e morale. Ma quanto resta nell'ombra, rispetto alla messa a fuoco abbagliante, tragica e anti-tragica allo stesso tempo, della figura di Socrate, che Platone cesella tra l'Apologia, il Critone e il Fedone? E quanto potremmo ancora discutere, restando vicini a Socrate, ma distogliendo gli occhi da un'immagine forse troppo pensata per l'eternità?

Domande di questo tipo devono aver attraversato la mente di Livio Rossetti, interprete originale e assai poco canonico di tutto ciò che gira intorno a Socrate nella fatidica Atene del V secolo, quando ha pensato di mettere in scena una pièce alternativa sulla fine del bizzarro ateniese (atopos per antonomasia), non più centrata sull'ironico eroismo del suo (platonico) saper morire, ma sulla cocciutaggine del suo vivere secondo coscienza, senza nulla concedere alle opinioni del più comune buon senso. Bisogna aver letto e sentito recitare il dialogo in tre atti Convincere Socrate (Diogene Multimedia, Bologna 2017) per rendersi conto che l'autore ha trovato molti buoni motivi per riscrivere le scene della sequenza drammatica che porta alla morte di Socrate. Lo ha fatto tenendo vivo il palinsesto dei dialoghi platonici, ma censurandolo, interpolandolo con scene laterali che allargano il contesto, introducendo sottili varianti di contenuto e di stile, che ci restituiscono una problematica significativamente diversa da quella suggerita da Platone.

Un Socrate meno filosofo, ma non meno pervicace nel difendere le sue convinzioni, ci parla dai due contesti principali che lo ritraggono in situazione pubblica, alla fine del processo, e in prigione, il giorno prima dell'esecuzione attesa, di fronte alla sua famiglia e ai suoi amici. Molto spazio autonomo ricevono le voci di coloro che non si rassegnano a perderlo, imputandogli in diversi modi la colpa di restare soltanto fedele a sé stesso e di non accogliere le ragioni della vita e dell'amore accanto a quelle dei principi per cui intende sacrificarsi. Una decisa innovazione investe poi il gruppo dei Socratici, che, smorzati i toni commossi e l'aura sacrale della rappresentazione platonica, appare ben 
diverso da un cenacolo intellettuale votato alla homologia, e piuttosto segnato da linee di frattura sociale, al di là del legame affettivo che unisce ciascuno al suo Socrate. Ma gli "scarti" dalla narrazione platonica proposti da Rossetti sono più numerosi e profondi, certo intenzionali e strategici.

La prima scena parte facendoci ascoltare solo la fine dell'intervento apologetico di Socrate, tagliando via, come inessenziale, ogni riferimento alla complessa orchestrazione platonica intorno all'immagine pubblica di questo filosofo di strada, dall'investitura ottenuta dall'oracolo delfico (Platone, Apologia 20d-22e) alla perorazione da lui rivolta al «cittadino di Atene» come destinatario politico della sua «missione per conto del dio» (Platone, Apologia 29d-30a). Il Socrate di Rossetti si rivolge alla buona ai suoi concittadini, richiamandosi alla loro coscienza per il giudizio sulla sua innocenza e rinunciando alla teatralità delle suppliche d'uso per «stima» nei loro confronti. Dopo la condanna, si difende ricordando di non aver ammazzato nessuno e di non essere certo «'sto gran pericolo per la città di Atene». E quando alla fine lancia la famosa sfida ai suoi giudici, dicendo di meritare un premio invece che una condanna, sembra dirlo per scherzo, con quell'enfasi ingenua che si usa per rimbeccarsi tra amici, mentre gli sfugge la portata reale della situazione in cui è stato coinvolto. Rossetti ha insomma calcato la mano sul minimalismo di questo Socrate, deciso a rintuzzare le accuse pretestuose di Meleto e a denunciarne in Anito il vero mandante, ma anche a ricondurre la causa di tanto risentimento a una questione privata: l'invidia di un padre per l'uomo che ha affascinato suo figlio (l'appoggio è in Senofonte, Apologia di Socrate 29-31 e il passo è riportato nell'Appendice del libretto).

Di contro, l'attenzione si sposta, a contrasto, sull'impersonalità procedurale delle norme che conducono all'esito mortale del processo a Socrate: l'Arconte Basileus gli toglie la parola perché il tempo dell'autodifesa è finito, si preoccupa di spiegare accuratamente, chiedendo la massima concentrazione, le norme e i dettagli tecnici della doppia votazione. Perché questo contrappunto? L'effetto è straniante, ma l'importanza che Rossetti attribuisce a questo richiamo alle norme processuali in uso nella città della democrazia (con tanto di documentazione riportata in Appendice, dove si spiega che cosa voleva dire «essere processati al tempo di Socrate») sembra suggerire una riflessione su quanto poco valgano le forme procedurali a garantire la sostanza della giustizia, se mancano vincoli etici profondi tra i cittadini e le istituzioni. Con buona pace di Kelsen et di Bobbio, sulla natura essenzialmente « procedurale » della democrazia (Bobbio, $I l$ futuro della democrazia, Einaudi, Torino 2005).

La seconda scena mette a fuoco il gruppo dei socratici, che troviamo impegnati a decidere il da farsi nella lussuosa casa di Critone. Vi sono in realtà riuniti solo i più ricchi di loro (tra cui Apollodoro, Adimanto e Platone), forti del ruolo sociale che li distingue e li fa ragionare secondo logiche di potere, ovviamente a sostegno di Socrate, $\mathrm{ma}$ anche in difesa della loro rispettabilità, messa alla prova dalla difficolta di salvarlo. Il danaro è al centro dei loro pensieri; e Rossetti ha la malizia di far arrivare il povero Eschine (il «pezzente» del gruppo) in ritardo, con l'inutile offerta della sua mezza mina, facendo apparire la sua povertà un simbolo di inadeguatezza mentale oltre che sociale. Noi tutti sappiamo già che la fuga dal carcere non potrà essere una soluzione per Socrate; ma vederne nascere la strategia dal conciliabolo dei Socratici più facoltosi rende subito difficile proiettare sul gruppo il modello di una coesione etica ideale.

La partita finale del dramma si gioca nella terza scena, che corrisponde, per situazione e contenuto, a quella del Critone e in piccola parte del Fedone platonici. Qui il dubbio sul valore reale dell'appartenenza o condivisione di principi, già emerso nell'area pubblica, si ripropone nel privato, dentro il più stretto entourage del filosofo. «Povera Euriclea, 
gli dei ti hanno tolto il senno», ripete Socrate più volte $(48,52)$, sbalordito di fronte alla proposta di un modo per salvarsi la vita che gli viene dai suoi più cari amici, ma che egli ritiene metterebbe fine a tutto ciò in cui crede, incrinando l'identità morale cui tiene più che alla vita. La stessa formula la usano anche Santippe (65) e Critone (68), ma per parlare di lui. La citazione omerica (è Penelope a pronunciare la frase, non dando credito, a torto, a quanto la nutrice Euriclea le dice sul ritorno di Ulisse) copre come un ombrello bucato le intenzioni diverse di coloro che la usano, attingendola a un patrimonio letterario comune. La frase commisera il pazzo, o chi appare tale agli occhi degli altri. Ma l'immagine della pazzia rimbalza dall'uno all'altro, tra persone che seguono ciascuna il proprio ragionamento e reciprocamente non si riconoscono. Pazzia è, agli occhi degli amici di Socrate, la sua cocciuta volontà di perseguire solo la coerenza con sé stesso; pazzia è, ai suoi occhi, abbandonare l'impegno a perseguirla in ogni azione e a portarla con sé fino ai giudici infernali, che, «se esistono», dovranno accoglierlo con una buona opinione di lui $(30,52,54,55-56,60-61)$.

La posta in gioco è la fuga in Tessaglia, come nel Critone platonico, ma qui non scendono in campo i Nomoi, a richiamare ai suoi doveri di cittadino il povero Socrate, condannato a morte con tutti i crismi della legalità. Qui il problema è che la coscienza di Socrate non tollera di praticare la menzogna e l'inganno, con l'aggravante dell'incongruenza di una tale scelta rispetto al fine di restare padrone della sua vita, nella sua città, fino all'ultimo minuto. Il segreto della resistenza di Socrate a ogni tentativo di convincerlo a una scelta opportunistica non sembra in sostanza risiedere in profonde convinzioni filosofiche, $\mathrm{ma}$ in una fedeltà al proprio modo di essere e alla forma di vita che ha scelto. Quest'uomo è certo un esempio di resistenza morale, ma appare davvero restio ad assumere l'abito del maestro.

Assai blanda appare poi, nel complesso, la preoccupazione di presentarsi senza macchia ai giudici dell'Aldilà: alla continuità tra questa vita e l'altra (di cui anche il Socrate platonico comunque dubitava) il Socrate di Rossetti non dedica niente di simile ai complessi (e fallaci) argomenti del Fedone sull'immortalità dell'anima, limitandosi a una ragionevole ma non necessaria «speranza» che tale continuità ci sia, consentendogli di essere ancora e sempre fedele a sé stesso.

Maliziosamente l'autore gli fa dire che forse solo Platone, assente dalla scena finale, avrebbe capito perché poteva accettare di rinunciare a un «pezzetto di futuro» pur di conservare sé stesso (78-79). Per quel pezzetto perduto Platone gli avrebbe in effetti regalato un'immortalità da filosofo, rappresentandolo nell'atto della sua instancabile ricerca dialogica fino all'ultimo respiro. Ma questo Socrate minimizza. E l'argomento cardine del Fedone, con cui il Socrate platonico dissocia l'anima dal corpo (79c-80a; 91c-92e), riconoscendole il potere di dominarlo e precederlo nel sapere (contro la tesi dell'anima-armonia: 85e-86d), diventa un'ipotesi nata per caso, ascoltando l'uomo della cicuta descrivere la lenta morte indotta dal veleno, a partire dai piedi (81-82): Apollodoro ne trae l'idea che l'anima è forse in grado di sfilarsi dal corpo come fanno «i serpenti» quando abbandonano la loro pelle; Socrate aggiunge argomenti a favore dell'autonomia decisionale dell'anima, ma senza impegnarsi affatto in dubbie dimostrazioni di immortalità (90-91). Di più: senza evocare il fantasma del Socrate naturalista e ateo di Aristofane, Rossetti gli fa tranquillamente usare gli strumenti osservativi e analogici ben noti all'indagine sulla natura del suo tempo.

Tirando le fila, la ragion d'essere della riscrittura di Rossetti mi sembra star tutta nel mostrare ciò che Platone nasconde, mentre fa di Socrate il modello del proto-filosofo e lo proietta come un gigante per theia moira nello scenario drammatico dell'Atene del v secolo. Rossetti sa bene dove trarre elementi per far vedere anche il resto. E scava, con 
tutta la sapienza dei suoi decenni di indagine socratica, nelle fonti meno canoniche, per restituire a Socrate il milieu in cui la sua esperienza di intellettuale irriverente ha concretamente preso forma: dentro i vincoli dell' ambiente a lui più vicino, nell'ambiguità dei ruoli familiari e sociali, nelle frequentazioni variegate e non necessariamente affidabili in cui è esplosa la provocazione del suo dialogare senza remore, tra gli amici che avrebbero fatto il possibile per trasmetterne l'eredità, ma che certo leggevano tutto a loro misura.

Leggendo, avevo in mente una diversa riscrittura: l'autentico «falso d'autore» di Mario Vegetti (Platone, Repubblica Libro XI, Lettera XIV, Guida Editore, Napoli, 2004, rist. 2018), in cui si gioca al ritrovamento di manoscritti perduti per dar vita al dialogo impossibile tra due grandi sconfitti della storia, Socrate e Marx, evocato come «Lo straniero di Treviri». Altri intenti, altre suggestioni; ma ancora Socrate, come figura di resistenza, che non smette di alimentare i nostri pensieri.

Quanto al valore delle riscritture, Platone sarebbe certo d'accordo che non c'è da fidarsi del tutto, perché $\mathrm{i}$ «giardini di scrittura» si seminano per gioco. Ma è davvero «un gioco bellissimo» quello di chi «è capace di giocare con i discorsi e di comporre storie sulla giustizia e sulle altre cose di cui tu [Socrate] parli» (Fedro 276d-e).

Fulvia De Luise

Università di Trento

Emmanuelle Jouët-PASTRÉ, Le plaisir à l'épreuve de la pensée. Lecture du Protagoras, $d u$ Gorgias et du Philèbe de Platon, Leiden-Boston, Brill, 2018 (Brill's Plato Studies Series), XII-293 p., ISBN HB : 978-90-04-36558-2 ; eBook : 978-90-0436559-9.

L'ouvrage prend pour objet la question complexe de la relation entre le plaisir et la pensée chez Platon. Il s'agit de montrer comment la pensée intelligente peut avoir prise sur le plaisir, mais également la façon dont cet illimité qu'est le plaisir peut résister jusqu'au bout à la philosophie. Dans cette mesure, la pensée met le plaisir à l'épreuve en se posant comme sa vérité, mais le plaisir met tout autant à l'épreuve la puissance de la pensée sur les âmes. Cette double problématique, qui entend dépasser les débats traditionnels sur l'hédonisme ou l'anti-hédonisme de Platon, guide l'étude des trois dialogues « dans lesquels le philosophe donne à penser le lien paradoxal et nécessaire entre plaisir et pensée » (p. 11): la fin du Protagoras (p. 15-56), la critique de l'hédonisme de Calliclès dans le Gorgias (p. 57-131), et enfin le Philèbe (p. 133-244), qui constituent autant de chapitres.

Le débat sur l'akrasia (incontinence) à la fin du Protagoras permet de mettre en évidence l'indissociabilité chez Platon du plaisir et d'une forme de logos : contre l'opinion qui l'explique comme une défaite de la raison face au plaisir, Socrate montre que l'hédonisme lui-même appelle, de par son caractère illusoire, une métrétique, une technique intelligente qui calcule plaisirs et peines et répond à l'humaine mesure protagoréenne. On a donc dans le Protagoras, comme c'est également le cas dans le Philèbe, « un dépassement de l'hédonisme à partir de ses propres critères » (p. 18): il y a toujours une forme de calcul dans la recherche du plaisir, et il s'agit moins d'y introduire de la raison que de lui conférer sa forme correcte en indiquant les conditions d'un bon calcul. On ne saurait donc faire droit à une interprétation trop strictement intellectualiste du refus de l'akrasia dans ce dialogue, ce qui conduit l'auteure à refuser également l'idée d'un remaniement doctrinal entre les premiers dialogues et la République, où d'une part la raison demeure indissociable des affects, et où d'autre part c'est seulement lorsque la 